\title{
AN EXPOSITION OF WIRELESS SENSOR NETWORK AREA COVERAGE AND LIFETIME BASED ON META HEURISTIC AND PARTICLE SWARM OPTIMIZATION ALGORITHM
}

\author{
ASIF FAROOQ ${ }^{1}$, TAHIR IQBAL ${ }^{2}$ \\ ${ }^{1}$ Department of Camputer Science, University of Lahore, Pakistan \\ ${ }^{2}$ Department of Camputer Science, Bahria University Lahore campus, Lahore, Pakistan \\ Email: asif.farooq@cs.uol.edu.pk, tahir.iqbal@bahria.edu.pk
}

\begin{abstract}
An important issue among the most vital and essential issues in Wireless Sensor Networks (WSNs) is the area coverage problem. This issue in WSNs causes the security situations directed by the current sensors in the systems suitably. The significance of scope in WSNs is important to the point that is one of the natures of administration parameters. In the event that the sensors don't suitably cover the physical situations they won't be sufficient proficient in supervision and controlling. The scope in WSNs must be in a manner that the vitality of the sensors would be the slightest to build the lifetime of the system. Alternate reasons which had expanded the significance of the issue are the topological changes of the system finished by the harm or cancellation of a percentage of the sensors and now and again the system should not lose its scope. Along these lines, in this paper we have half and half algorithm, the Meta-Heuristic calculations like Differential Evolution and Particle Swarm Optimization algorithms and have broken down the range scope issue in WSNs. Additionally PSO algorithm is executed to look at the productivity of the half and half model in the same circumstances. The consequences of the trials demonstrate that the half and half algorithm has made more increment in the lifetime of the system and more upgraded utilization of the vitality of sensors by improving the scope of the sensors in comparison to PSO.
\end{abstract}

Keywords - Network Security, Algorithms, Area coverage, Sensor

1. Introduction:Wireless Sensor Networks are being used widely in the fields of business, research and operation. The WSNs contain numerous sensors which are appropriate in the management and security environments [1].WSNs is clever to manage the expected environments to control and process the collected information. In WSNs it is essential to study the coverage and energy use problems to enhance the lifespan of the network for sending data and lifespan of the network would not face extensive reduction [2].The most important features of designing the WSN are coverage of network and use of energy. By giving the environmental situation of these networks, it is not conceivable to replace the battery of the thousands of the sensors $[3,4]$. So, the problem of coverage in WSNs is directly related to the increase of the lifespan of the sensors. The best situation for the WSNs is the time that all nodes are located in an appropriate sensor radius distance. And this means that the network has extensive lifespan [5]. So, to enhance the lifespan of the network, the sensor circulation must be balanced.

The most significant factor for mounting and scaling of the WSNs is to contemplate the problem of area coverage and reducing the usage of energy of the sensing devices [6]. The coverage must be in such a way that all those sensors which are active must be able to cover all points and also the least number of the sensors will be used and coverage area must be set according to the size of the nearest sensors and distance to the nearest sensor is required for sensing and transferring the information. The key role of covering area in WSNs is to supervise surroundings totally [7]. As shown in figure below any point below the coverage surroundings must be enclosed at least by one sensor. The perfect area coverage occurs when we cover desired area completely by using minimum number of sensing devices [8] [9]. 

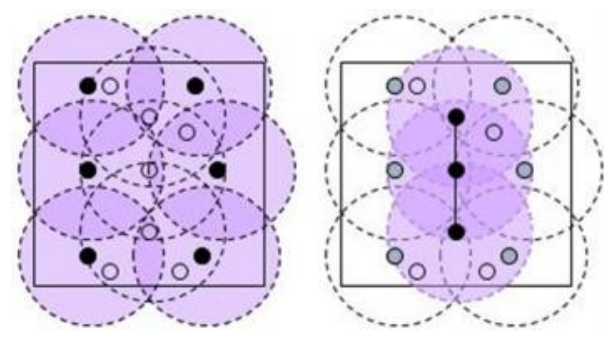

Figure 1: Area Coverage

2. Related Work: In wireless communication field one of the most important area of research is WSN. A set of sensors is included in WSN which are dispersed in supervising settings and then obtained data is processed and finally the base station is provided with the desired statistics. A lot of approaches are vacant for cultivating the problem of coverage and its quality in WSNs till now.

In [10] Multi-Objective Particle Optimization (MPSO) is used in coverage problem for energy efficiency and increase in lifespan of WSNs is also studied. The main objective of MPSO is to discover the best location for the sensors for improved coverage. According to the consequences of the trials it is probable to say that MPSO is proficient in coverage and enhancing the lifespan of the network. In [11] the author described K-coverage problem in WSNs by the use of Harmony Search (HS). In this reference it is supposed that the two most important parameters for wireless sensor nodes distribution are coverage and energy efficiency. HS algorithm is used for improved cooperative sensors ad minimizing the energy use. In K-coverage the area is enclosed by k sensors. The results of the trials in this reference show that the HS algorithm is very efficient in coverage and minimizes the energy use of the sensors. In [12] the author proposed a novel approach of distributed algorithm with effectual energy use and optimized coverage. In proposed algorithm the sensors are divided into active and inactive divisions and produce a graph model which clues to the stable energy use of the sensors. In this reference, only the active sensors are used for coverage and the rest of the sensors go deactivated. Turning off the unnecessary and redundant sensors, the other sensors of the network use less energy and as a result the lifespan of the network increases. The results of the simulations show that the efficiency of the suggested algorithm is more than the under comparison algorithms and it is able to increase the lifetime of a network up to multi times more. In [13] the author describes the positioning technique of the sensors in WSNs according to the point coverage. This technique is shaped by some goal points, the formation of the sensors is very affective on the number of the required sensors for coverage of the points. According to authors that technique the point coverage occurs according to the location of the goal and angle of the goal points. In [14] the author used Fish Swarm (FS) and PSO algorithms hybrid to discuss the WSNs coverage problem. PSO algorithm is used in hybrid algorithm for more efficiency and FS for covering the sensors. The results show that the hybrid algorithm is efficient enough in deployment of the sensors of network and has improved the coverage problem. In [15] the sensing coverage of any sensor is set in a definite space for growing the effectiveness of the energy of the sensors. Two heuristic algorithms are used by the author for the results of the experiences. The outputs of the trials show that Greedy is more efficient in energy consumption and network coverage as compared to Linear Programming. In [16] the author proposed a heuristic algorithm to cover entirely a region having arbitrary (opaque) problems which allow neither the sensor to be placed inside nor the signals to pass through. Firstly the author deploy an optimal design for covering a plane over the region, and then locate and competently cover the uncovered dumps designed by the obstacles. In [17] the author proposed a dynamic algorithm for area coverage problem in WSNs. The most important problem in coverage is the distribution of the sensors and the lifespan of the network. According to author the sensors are distributed according to the command area and the shape is most important creation. The sensors can also have vigorously topological changes in the atmosphere. The most significant provisions measured in this reference is the use of energy by sensors. The experimental results shows that the proposed algorithm is more efficient than the other algorithms from establishment of the sensors point of view. In [18] the author used Ant Colony Optimization (ACO) Algorithm in WSNs coverage problem. The perfect radius for the sensors is recognized by using ACO algorithm. When it is required to have an active network for long time and the sensing limits of the any sensor is identified the use of ACO algorithm is appropriate. The author estimated the improvement of the energy use problem in sensor networks using the area coverage and have cleared that ACO is competent in lifespan of network. 
According to the results of their simulation, ACO algorithm is efficient in lifespan of the network. In [19] the author have used the heuristic algorithms for the coverage problem in large scales. For optimization of coverage problem in bulky scales the Greedy algorithm is utilized. The author's goal is to use the heuristic algorithms to establish the sensors in the points of the spaces which cover the network in the best manner and have the best lifespan. Any point could have many terminated sensors and if they are active more energy is used. So, the sensors which are not used are disabled by the Greedy algorithm a just the sensors which are in relation to the base station are activated. The experimental results show that the Greedy algorithm is the best solution for coverage problem of the sensors in large scale. In [20] the author proposed a new protocol based on the dynamic structure of the sensors for coverage problem. In his proposed protocol, the sensors are activated or deactivated for energy saving. Also, the position of the sensors is dynamically identified and any sensor can cover its radius well. They have proved that the propose algorithm is more efficient in coverage.

3. Technical Framework: In this section different techniques and algorithms for solving area coverage problem in wireless sensor network will discussed in details technically.

\section{Meta-Heuristic Algorithms:}

Meta-Heuristic Algorithms are in light of populace to the streamlining issues and are devices for discovering the close to improved arrangements. These calculations use the differing qualities and participation ideas and improve the advancement space in accomplishing the most streamlined status. In this way, the more the force of a calculation in controlling the two parameters, the more calculations is able in discovering the most improved stage. In this area we discuss PSO and DE algorithms which are the most essential populace Algorithm.

\section{Particle Swarm Optimization:}

PSO is a reproduction of the social conduct of the winged creatures which hunt down nourishment in and environment. None of the winged animals have data about the spot of the sustenance yet they know in every stage how far they are from the nourishment. On this premise, the best methodology to discover nourishment is to take after the closest fledgling to the sustenance.

PSO calculation is a populace calculation in which various the particles which are the answers for a capacity or issue shape a populace. A populace of the particles moves in the issue space and tries to locate the most improved reply in the seeking space as indicated by their own particular experience furthermore the populations. PSO Algorithm is an improved algorithm which gives a hunt in light of the populace in which any molecule changes its position by the time. In PSO algorithm the particles move in a looking space of multi measurement including the conceivable arrangements. In this space an assessment element is characterized and the quality assessment of the arrangements of the issue happens by it. Any change of a molecule in a gathering is influenced by the self or other's experience and the looking conduct of a molecule is influenced by the other particles. This basic conduct reasons discovering upgraded ranges of the seeking space. Along these lines, in PSO algorithm any molecule which discovers the streamlined circumstance, illuminates alternate particles in a suitable way and any molecule chooses for the expense capacity as indicated by the accomplished qualities what's more, seeking happens utilizing the ex-learning of the particles. This cause the particles don't get close to one another more than the typical and take care of the enhancement issue viably. In PSO algorithm, first the gathering individuals are made arbitrarily in issue space and the scanning procedure for the streamlined answer begins. In the aggregate structure, the pursuit of any part takes after the other which is the most streamlined suitable estimation of the capacity and it doesn't overlook its experience and takes after the state in which he suitability capacity worth was the most for itself. Along these lines, in every reiteration, any part changes its circumstance as per the two values, one of them is the best circumstance of the part till then (pbest) and the other is the best circumstance the aggregate populace has had till then. In fact it is the pbest in the total population (gbest). In perception, pbest for any member is in fact the biologic memory of the member. gbest is the general knowledge of the population and when the members change their situation according to gbest, in fact they try to heighten the knowledge level up to the general knowledge of the population. From the concept view, the best particle of the group relates all other particles to each other. The credentials of the next location of any particle is done by using equations (1) and (2)

$$
\begin{gathered}
u_{k+1}=x \cdot m_{i}+d_{1} \cdot r_{1} \cdot\left(Q_{\text {best }_{i}}-x_{i}\right)+c_{2} \cdot r_{2} \cdot\left(h_{\text {best }_{i}}-x_{i}\right) \\
y_{i+1}=x_{i}+v_{i+1}
\end{gathered}
$$


In equation (1) $c_{1}$ and $c_{2}$ are the learning parameters. $x_{i}$ is the existing condition and $v_{i}$ is the moving speed of the associates. W is a control parameter which the current speed $v_{i}$ with the next one and produce the balanced state among the aptitude of the algorithm in local and global searching and then extents the answer in shortest time. For optimized operation of algorithm in searching space parameter $\mathrm{W}$ is introduced as

$$
\mathrm{Z}=\mathrm{z}_{\max }-\frac{\left(\left(\mathrm{z}_{\max }-\mathrm{z}_{\min }\right) \times \mathrm{j}\right)}{\mathrm{j} \max }
$$

In this equation $j_{\max }$ shows the maximum number of repetition of the algorithm and the parameter $i$ is the counter of repetition. $Z_{\max }$ and $z_{\min }$ are the primary value and final value of inertia weight in algorithm execution time.

Differential Evaluation: DE is population based algorithm and probable for searching. DE uses the distance and direction from the current population information to endure searching. Speed, efficiency in finding optimized solution, parameter setting and no need of ordering and matrix coefficient are some major advantages of DE algorithm. DE algorithm is intelligent to search in direction of the coordinates of the optimization variables and altering the coordinates for finding the optimized solution. DE algorithm appraises the Searching technique from an arbitrary initial population. DE algorithm following stages:

Primary population: The solution vectors or the primary population was selected arbitrarily from the domain of issue in DE algorithm. The solutions position vector is presented by equation (4).

$$
\begin{gathered}
Y_{i j}=\left(y_{j, 1}, y_{j, 2}, \ldots \ldots \ldots y_{j, D}\right) \\
y_{j l}=y_{k}^{\min }+\operatorname{rand}(0,1) \cdot\left(y_{k}^{\max }-y_{k}^{\min }\right. \\
\text { With } \\
i \in\left[1, L_{q p}\right], k \in[1, D]
\end{gathered}
$$

In above equation $y_{j l}$ represents the selection of random number and $\mathrm{D}$ is denoting the dimensions of the solution. $L_{q p}$ is the number of initial population.

Mutation: Three random vectors were selected in mutation stage which all different. A new answer was calculated in each reappearance for any $\mathrm{j}$ vector in population using equation (6).

$$
u_{j, H+1}=y_{r_{1}, H}+\text { F. G }\left(y_{r_{2}, H}-y_{r_{3}, H}\right)
$$

In this equation $r_{1}, r_{2}$ and $r_{3}$ are the three randomly selected vectors and their values is different from each other and are located in the domain $\left[1, \mathrm{~N}_{\mathrm{p}}\right]$.Here $\mathrm{F}$ is a constant and $\mathrm{G}$ shows the generations produced.

Crossover: Crossover operator grounds growth in variety of the population. This operator is comparable with the crossover operator in genetic algorithm [39]. A new vector is created by hybrid of the $x$ and $v$ vectors in this operator as show in equation below (7)

$$
c_{k l, H+1}=\left\{\begin{array}{l}
d_{k l, H+1} \\
e_{k l, H+1}
\end{array} \text { if }\left(m_{k} \leq C R\right) \text { or } k=k_{\text {rand }}\right.
$$

In above equation CR is located in $[0,1]$. Parameter $m_{k}$ is created randomly in $[0,1]$ and the value of $\mathrm{k}$ is $1,2, \ldots \ldots$ D.

Selection: To choose the vectors of highest propriety from the vectors which are created by mutation and crossover operators are compared with each other and any of them having more correctness is moved to the next group. Following equation represents the selection operator

$$
y_{j, H+1}=\text { Fitness Value }\left(c_{l, H+1}, y_{i, H}\right)
$$

Stop: The searching process continues till the stopping factor of the algorithm is met. Usually the stopping facto of the algorithm could be based on the non-changing propriety of the best answer or algorithm repetition.

4. Comparative Study: This section is about the comparison of different techniques and procedures 
which are used for solving the problem of area coverage in wireless sensor network.

The Effect of the Number of the Sensors on the Lifetime of the Network:

In this section the effect of the number of the sensors on the lifetime of the network is considered. The radius area of any sensor is considered as 40. As it is clear from Figure (2) the lifetime of the network in hybrid algorithm linearly increases by the size of the networks.

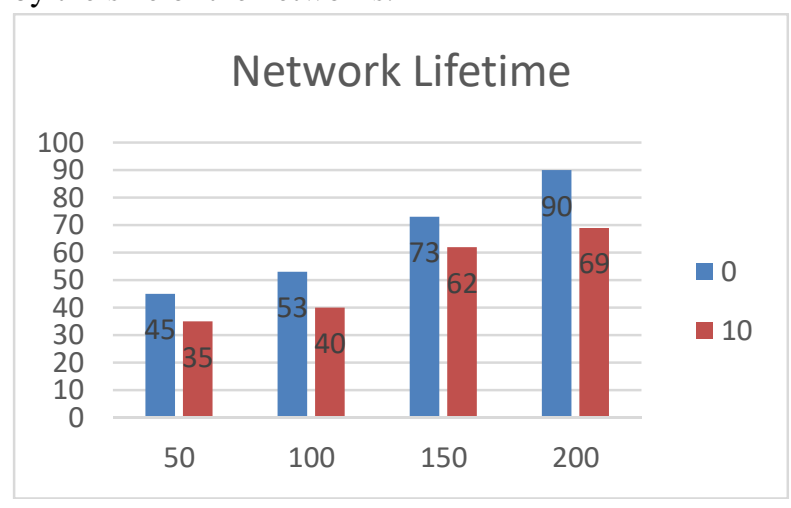

Figure 2: Estimation of lifespan of Network

The Comparison of the Sensor Coverage Percent:

Figure 3 displays the coverage percent comparison of hybrid and PSO algorithms. It can be seen in figure as the hybrid algorithm is more able to set the sensing area of the sensors it covers more efficient.

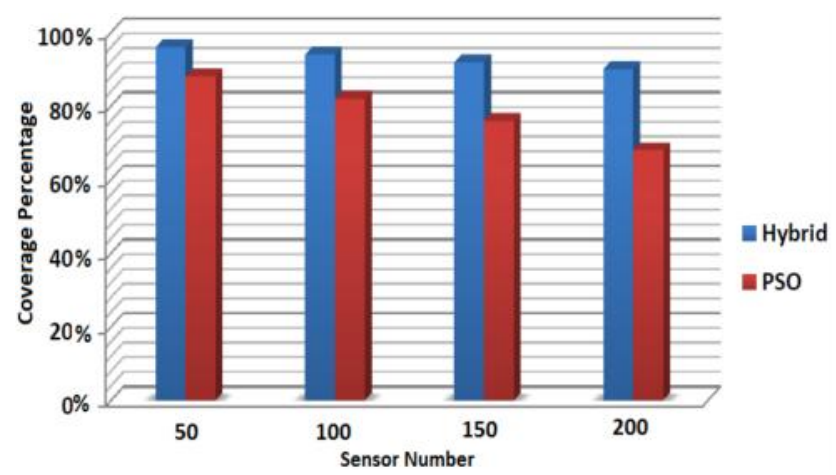

Figure 3: Efficiency comparison of Hybrid and PSO

5. Conclusion: Coverage issue is a standout among the most vital exploration fields of WSNs. In this paper we have utilized the PSO and DE algorithms for territory scope in WSNs and to expand the lifetime of the system. Consequently in crossover algorithm the two elements of suitable circulation of the sensors and the vitality diminish which prompt expanding the lifetime of the system utilization are considered. What's more, to demonstrate the productivity of the mixture algorithm better, it is contrasted with PSO algorithm and as per the aftereffects of the reproductions, it can be said that the crossover algorithm is better.

\section{REFERENCES}

[1] Rebai, M., Snoussi, H., Khoukhi, L., \& Hnaien, F. (2013, April). Linear models for the total coverage problem in wireless sensor networks. In Modeling, Simulation and Applied Optimization (ICMSAO), 2013 5th International Conference on(pp. 1-4). IEEE.

[2] Kuila, P., \& Jana, P. K. (2014). Energy efficient clustering and routing algorithms for wireless sensor networks: Particle swarm optimization approach. Engineering Applications of Artificial Intelligence, 33, 127-140.

[3] Yick, J., Mukherjee, B., \& Ghosal, D. (2008). Wireless sensor network survey. Computer networks, 52(12), 2292-2330.

[4] Zhu, C., Zheng, C., Shu, L., \& Han, G. (2012). A survey on coverage and connectivity issues in wireless 
sensor networks. Journal of Network and Computer Applications, 35(2), 619-632.

[5] Jain, E., \& Liang, Q. (2005). Sensor placement and lifetime of wireless sensor networks: theory and performance analysis. In Global Telecommunications Conference, 2005. GLOBECOM'05. IEEE (Vol. 1, pp. 5-pp). IEEE.

[6] Chen, Z., Li, X., Lv, B., \& Jia, M. (2015, August). A Self-Adaptive Wireless Sensor Network Coverage Method for Intrusion Tolerance Based on Particle Swarm Optimization and Cuckoo Search. In Trustcom/BigDataSE/ISPA, 2015 IEEE(Vol. 1, pp. 1298-1305). IEEE.

[7] Tan, H., Hao, X., Wang, Y., Lau, F. C., \& Lv, Y. (2013). An approximate approach for area coverage in wireless sensor networks. Procedia Computer Science, 19, 240-247.

[8] Alam, K. M., Kamruzzaman, J., Karmakar, G., Murshed, M., \& Azad, A. K. M. (2011). QoS support in event detection in WSN through optimal k-coverage. Procedia Computer Science, 4, 499-507.

[9] Yu, J., Deng, X., Yu, D., Wang, G., \& Gu, X. (2013). CWSC: Connected k-coverage working sets construction algorithm in wireless sensor networks. AEU-International Journal of Electronics and Communications, 67(11), 937-946.

[10] Chaudhary, D. K., \& Dua, R. L. (2012). Application of multi objective particle swarm optimization to maximize coverage and lifetime of wireless sensor network. Int. J. Comput. Eng. Res, 2, 1628-1633.

[11] Nezhad, S. E., Kamali, H. J., \& Moghaddam, M. E. (2010, November). Solving K-coverage problem in wireless sensor networks using improved harmony search. In Broadband, Wireless Computing, Communication and Applications (BWCCA), 2010 International Conference on (pp. 49-55). IEEE.

[12] Dhawan, A., \& Prasad, S. K. (2009). A distributed algorithmic framework for coverage problems in wireless sensor networks. International Journal of Parallel, Emergent and Distributed Systems, 24(4), 331-348.

[13] Fan, G., Liang, F., \& Jin, S. (2008, August). An efficient approach for point coverage problem of sensor network. In Electronic Commerce and Security, 2008 International Symposium on (pp. 124-128). IEEE.

[14] Bin, Z., Jianlin, M., \& Haiping, L. (2011, March). A hybrid algorithm for sensing coverage problem in wireless sensor netwoks. In Cyber Technology in Automation, Control, and Intelligent Systems (CYBER), 2011 IEEE International Conference on (pp. 162-165). IEEE.

[15] Cardei, M., Thai, M. T., Li, Y., \& Wu, W. (2005, March). Energy-efficient target coverage in wireless sensor networks. In INFOCOM 2005. 24th annual joint conference of the ieee computer and communications societies. proceedings ieee(Vol. 3, pp. 1976-1984). IEEE.

[16] Tan, H., Wang, Y., Hao, X., Hua, Q. S., \& Lau, F. C. (2010, August). Arbitrary obstacles constrained full coverage in wireless sensor networks. In International Conference on Wireless Algorithms, Systems, and Applications (pp. 1-10). Springer, Berlin, Heidelberg.

[17] Quintao, F. P., Nakamura, F. G., \& Mateus, G. R. (2005, September). Evolutionary algorithm for the dynamic coverage problem applied to wireless sensor networks design. In Evolutionary Computation, 2005. The 2005 IEEE Congress on(Vol. 2, pp. 1589-1596). IEEE.

[18] Liao, W. H., Kao, Y., \& Wu, R. T. (2011). Ant colony optimization based sensor deployment protocol for wireless sensor networks. Expert Systems with Applications, 38(6), 6599-6605.

[19] ali Jamali, M., Bakhshivand, N., Easmaeilpour, M., \& Salami, D. (2010, July). An energy-efficient algorithm for connected target coverage problem in wireless sensor networks. In Computer Science and Information Technology (ICCSIT), 2010 3rd IEEE International Conference on (Vol. 9, pp. 249-254). IEEE.

[20] Wang, X., Xing, G., Zhang, Y., Lu, C., Pless, R., \& Gill, C. (2003, November). Integrated coverage and connectivity configuration in wireless sensor networks. In Proceedings of the 1st international conference on Embedded networked sensor systems (pp. 28-39). ACM.

[21] Han, G., Liu, L., Jiang, J., Shu, L., \& Hancke, G. (2017). Analysis of energy-efficient connected target coverage algorithms for industrial wireless sensor networks. IEEE Transactions on Industrial Informatics, 13(1), 135-143.

[22] Njoya, A. N., Thron, C., Barry, J., Abdou, W., Tonye, E., Konje, N. S. L., \& Dipanda, A. (2017). Efficient scalable sensor node placement algorithm for fixed target coverage applications of wireless sensor networks. IET Wireless Sensor Systems, 7(2), 44-54.

[23] Mini, S., Udgata, S. K., \& Sabat, S. L. (2014). Sensor deployment and scheduling for target coverage problem in wireless sensor networks. IEEE Sensors Journal, 14(3), 636-644. 
[24] Mini, S., Udgata, S. K., \& Sabat, S. L. (2014). Sensor deployment and scheduling for target coverage problem in wireless sensor networks. IEEE Sensors Journal, 14(3), 636-644. 